Ann. Génét. Sél. anim., I974, 6 (2), I77-I86.

\title{
CONSÉQUENCES \\ DE L'HYPERTROPHIE MUSCULAIRE HÉRÉDITAIRE DES BOVINS SUR LA MUSCULATURE (1)
}

\author{
R. BOCCARD et B. L. DUMONT* \\ avec la collaboration technique de A. TALmant \\ Station de Recherches sur la Viande, \\ Centre de Recherches de Clermont-Ferrand, I. N.R. A., \\ Theix, 63110 Beaumont \\ * Laboratoire de Recherches sur la Viande, \\ Centre national de Recherches zootechniques, I. N. R. A., \\ 78350 Jouy en Josas
}

\section{RÉSUMÉ}

La musculature des demi-carcasses de 9 taurillons hypertrophiés $(\mathrm{H})$ et $\mathrm{I} 7$ taurillons normaux $(\mathrm{N})$ de race Charolaise de ro à 20 mois a été disséquée. Par calcul de régression les poids des principaux muscles ont été estimés pour une masse totale de muscle de $140 \mathrm{~kg}$ dans la demi-carcasse.

La comparaison entre les deux types a été faite sur la base du rapport $\frac{\mathrm{H}-\mathrm{N}}{\mathrm{N}} \times$ roo, où $\mathrm{H}$ représentait le poids d'un muscle ou d'un ensemble régional de muscles dans le type hypertrophié et $\mathrm{N}$ les éléments correspondants dans le type normal.

A poids de muscle constant l'hypertrophie affecte uniquement les régions crurales $(+9,20$ p. IOO), thoraciques $(+6,77$ p. I00) et brachiales $(+5,02$ p. I00); les autres régions sont retardées dans leur développement à des degrés divers.

Les modifications constatées dans la répartition des régions expliquent les différences d'aspect et de conformation des animaux sur pied. Le développement relatif des principaux muscles montre une très large variation.

L'hypertrophie affecte particulièrement les muscles de la cuisse (Tensor fasciae latae: $+20,3 \mathrm{I}$; Vastus lateralis : + 13,98 ; Semimembranosus : + 14,62; Biceps temoris : + I I,68; Semitendinosus : $+9,99 ;$ Rectus femoris : $+7,45 ;$ Gracilis : + 5,23; Pectineus : $+5,44$ p. roo) et, dans les autres régions, une partie seulement des muscles situés en plan superficiel (Latissimus dorsi : + 17,34; Pectoralis profundus: + 15,59; Triceps brachii caput laterale: + 16,67; et Trapezius: $+7,98$ p. I00).

Chez les animaux hypertrophiés certains muscles ont un développement relatif moindre : les muscles de la paroi abdominale, certains muscles de l'encolure, ce qui est en relation avec l'état d'immaturité sexuelle de ces animaux, et les muscles intervenant dans les phénomènes respiratoires (Diaphragma : — I 7,97 et région thoracique costale : - I I,93 p. Ioo).

La teneur en collagène de muscles d'animaux hypertrophiés est nettement moindre que celle des muscles des animaux normaux ; de plus elle est indépendante de leur degré de développement.

(1) Cette étude a fait l'objet de brèves communications aux Journées d'Études de la F. E. Z. à Versailles en juillet I97I (Ann. Génét. Sél. anim., I972). 
Une hypothèse des causes de l'hypertrophie musculaire est proposée :

L'hypertrophie musculaire périphérique semble être la conséquence du déficit généralisé en collagène qui entraîne une sous-tension au niveau de la musculature. Le déficit en collagène serait lui-même la conséquence des moindres capacités respiratoires qui caractérisent les animaux hypertrophiés.

\section{INTRODUCTION}

L'hypertrophie musculaire héréditaire des bovins a donné lieu, depuis un siècle, dans diverses races, à des observations de différents ordres (LAUVERGNE et al., I963). La morphologie du caractère a été abondamment décrite, les aspects concernant la composition anatomique ont été moins étudiés et de façon plus récente. VIssac (I968) en particulier, a considéré la composition de la carcasse en morceaux isolés selon le processus d'une découpe commerciale de lots d'animaux Charolais "culards" et normaux à trois âges différents. Par ailleurs les travaux intéressants la répartition et l'importance relative des muscles n'ont porté que sur des individus isolés, comparés à des sujets normaux dont le poids de la musculature était différent de celui des sujets hypertrophiés (BuTTERFIELD, I966 ; Rollins et al., I969).

Le présent travail rapporte les résultats d'une étude de la distribution de la musculature et de la composition des muscles, à un poids total de muscle identique, chez les bovins manifestant une hypertrophie musculaire (caractère " culard ") et chez des sujets de même race et de type morphologique normal.

\section{MATÉRIEL E'T MÉTHODES}

\section{Étude anatomique}

L'étude a porté sur des taurillons de race Charolaise. On a considéré $\mathbf{1} 7$ animaux de type normal dont l'âge allait de Io à 20 mois, et 9 animaux de type hypertrophié de 14 à 20 mois d'âge. Les poids moyens de carcasse chaude étaient de $329,4 \pm 64,7 \mathrm{~kg}$ pour les animaux normaux et de $378,2 \pm 47,9 \mathrm{~kg}$ pour les sujets hypertrophiés. Le lendemain de l'abattage on a procédé à la dissection d'une demi-carcasse. A partir des poids de muscles obtenus on a calculé par addition les masses représentées par les muscles des diverses régions du corps mentionnées au tableau I. Par la méthode des moindres carrés on a établi les équations de régression du poids de ces ensembles de muscles sur le poids de la musculature totale. A partir des relations obtenues on a estimé le poids moyen attribuable à ces groupes de muscles lorsque le poids de la musculature de la demi-carcasse était de $140 \mathrm{~kg}$, poids moyen choisi en fonction des valeurs observées dans les deux groupes d'animaux examinés. On a procédé de la même manière pour les principaux muscles considérés individuellement et mentionnés au tableau 2. Une méthode semblable de calcul a été employée pour estimer la masse de l'ensemble du squelette et celles de ses principaux éléments. Pour les régions musculaires et les muscles on a calculé les valeurs du rapport $\frac{\mathrm{H}-\mathrm{N}}{\mathrm{N}} \times$ roo, où $\mathrm{H}$ est le poids de la région (ou du muscle) du type hypertrophié, et $\mathrm{N}$ le poids de l'élément correspondant dans le type normal.

Ce rapport traduit le degré de développement massique des muscles hypertrophiés par rapport à ceux du type normal. Quand le rapport est supérieur à o il y a lieu de parler d'une réelle hypertrophie du muscle ou de l'ensemble des muscles considérés. Quant le rapport est inférieur à o, on a affaire à une hypotrophie relative. Lorsque le rapport est nul il y a similitude de développement massique des muscles chez les deux types d'animaux. 


\section{Étude chimique}

Chez quatre taurillons de 15 mois, deux de type hypertrophié et deux de type normal, on a procédé sur treize de leurs muscles, après prélèvement au scalpel et élimination de leur épimysium, à la détermination de la teneur en azote total et de la teneur en hydroxyproline selon la méthode précédemment employée (BOCCARD, 1967).

Le rapport $\frac{\text { azote de l'hydroxyproline }}{\text { azote total }} \times \mathrm{IO}^{3}$ a été retenu pour exprimer la teneur en collagène des muscles.

\section{RÉSULTATS E'T DISCUSSIONS}

\section{Étude anatomique, répartition des muscles}

Le tableau I indique le poids des ensembles de muscles de diverses régions, pour le type normal et pour le type hypertrophié, ainsi que les valeurs du rapport relatif du type hypertrophié vis-à-vis du type normal $\left(\frac{\mathrm{H}-\mathrm{N}}{\mathrm{N}}\right) \times$ 100). Le tableau 2 mentionne les données correspondant aux principaux muscles, classés par ordre de valeur d'hypertrophie massique décroissante.

\section{TABLEAU I}

Masse (en g), des ensembles de muscles des diverses régions de la demi-carcasse (à $140 \mathrm{~kg}$ de muscle)

\begin{tabular}{|c|c|c|c|c|}
\hline Régions musculaires & Type & $\begin{array}{c}\text { Type } \\
\text { hyper- } \\
\text { trophié }(\mathrm{H})\end{array}$ & $\frac{\mathrm{H}-\mathrm{N}}{\mathrm{N}}$ & $+\times 100$ \\
\hline & & & & \\
\hline Muscles du cou $\ldots \ldots \ldots \ldots \ldots$ & 17878,5 & 16749,5 & - & 6,31 \\
\hline Muscles du membre antérieur..... & 18774,2 & 18632,2 & - & 0,76 \\
\hline Muscles de l'avant-bras $\ldots . . \ldots$ & 3330,3 & 3335,5 & + & 0,16 \\
\hline Muscles du bras $\ldots \ldots \ldots \ldots \ldots$ & 7226,1 & 7588,9 & t & 5,02 \\
\hline $\begin{array}{c}\text { Muscles de la région scapulaire } \\
\text { interne } \ldots \ldots \ldots \ldots \ldots \ldots \ldots \ldots\end{array}$ & 2384,9 & 2214,0 & - & 7,17 \\
\hline $\begin{array}{c}\text { Muscles de la région scapulaire } \\
\text { externe } \ldots \ldots \ldots \ldots \ldots \ldots \ldots\end{array}$ & 5790,4 & 5493,8 & - & 5,12 \\
\hline Muscles de la région rachidienne... & 19837,7 & 18160,2 & - & 8,46 \\
\hline Muscles du thorax $\ldots \ldots \ldots \ldots$ & 17877,4 & 19087,5 & + & 6,77 \\
\hline $\begin{array}{c}\text { Muscles de la région diaphragma- } \\
\text { tique } \ldots \ldots \ldots \ldots \ldots \ldots \ldots\end{array}$ & 885,3 & 725,5 & & 18,05 \\
\hline Muscles de l'abdomen ......... & 14317,8 & 13482,0 & - & 5,84 \\
\hline $\begin{array}{c}\text { Muscles de la région abdominale } \\
\text { inférieure } \ldots \ldots \ldots \ldots \ldots \ldots \ldots\end{array}$ & 10313,5 & 9565,5 & - & 7,25 \\
\hline Muscles de la région sous-lombaire. & 4004,2 & 3917,1 & 一 & 2,18 \\
\hline Muscles de la fesse $\ldots \ldots \ldots \ldots$ & 6139,0 & 5866,4 & - & 4,44 \\
\hline Muscles de la cuisse $\ldots \ldots \ldots \ldots$ & 34606,1 & 38039,7 & + & 9,92 \\
\hline $\begin{array}{l}\text { Muscles de la région crurale posté- } \\
\text { rieure } \ldots \ldots \ldots \ldots \ldots \ldots \ldots \ldots\end{array}$ & 19555,6 & 21960,6 & +1 & 12,30 \\
\hline $\begin{array}{l}\text { Muscles de la région crurale anté- } \\
\text { rieure } \ldots \ldots \ldots \ldots \ldots \ldots \ldots \ldots\end{array}$ & 9718,8 & 10492,0 & + & 7,96 \\
\hline Muscles de la région crurale interne. & 5328,9 & 5587,1 & + & 4,85 \\
\hline Muscles de la jambe........... & 6320,4 & 5913,3 & 一 & 6,44 \\
\hline
\end{tabular}


$\mathrm{Au}$ niveau des régions musculaires on constate de nombreuses différences entre les deux types d'animaux. La dissemblance dans la répartition des groupes musculaires constitue une exception à la loi d'harmonie anatomique (BOccARD et Dumont, I960). D'autre part l'inégalité de l'importance relative des diverses régions est en contradiction avec les conclusions de différents auteurs (PoMERoY et WILLIAMs, I962 ; ButTERFIELD, I966 ; Rollins et al., I969; MCKELlar et OuHAYOUN, I973) qui ont avancé l'hypothèse de l'existence d'une hypertrophie musculaire généralisée. Cette contradiction apparente avec nos résultats résulte du mode de comparaison adopté pour apprécier la composition musculaire régionale relative des deux types d'animaux, qui ont été considérés, antérieurement, soit au même âge (BUTTERFIELD, I966), soit au même poids vif vide (Rol.LINs et al., I969). Quand elles s'établissent sur de telles bases les comparaisons entre types ne se font pas alors réellement entre animaux comparables sur le plan musculaire. En effet, en raison des différences de vitesse de croissance corporelle, les poids vifs vides enregistrés au même âge peuvent être très différents. En outre, à poids vif vide équivalent, les différences de rendement en carcasse conduisent à des carcasses plus lourdes chez l'animal hypertrophié. Enfin, même si l'on considérait les deux types d'animaux à poids de carcasse semblable, les différences dans la composition tissulaire relative font que les types hypertrophiés, plus musclés et moins gras, auraient des poids de musculature plus élevés. Or les différences de coefficients d'allométrie des divers muscles entraînent, à poids de musculatures différents et à poids de carcasses différents, des différences dans la répartition des muscles dont le calcul reposant sur le simple pourcentage ne tient pas compte (ANGERVAL et CARLSTROM, I963). Cette observation justifie la base de jugement retenue dans cette étude (poids de musculature identique) pour comparer les deux types de bovins.

Nos résultats conduisent à des valeurs du rapport du poids des muscles de la cuisse au poids des muscles du cou de 2,27 pour le type hypertrophié et de I,94 pour le type normal. Les rapports du poids des muscles de la cuisse à ceux de la région abdominale inférieure sont respectivement de 3,98 et de 3,36.

La différence de répartition de la masse musculaire totale entre les diverses régions explique, en partie, les différences d'aspect et de conformation qui séparent les deux types d'animaux. Le type "hypertrophié " se caractérise, on le sait, par la finesse de son encolure, par son ventre levreté et par le développement de sa cuisse. Ce caractère qu'on a récemment proposé d'apprécier visuellement à l'aide de standards de profils sur les sujets vivants (RoLlins et al., I972) pourrait être estimé quantitativement post mortem et pourrait être combiné au moindre développement de l'encolure ou de la région abdominale inférieure pour définir, au plan de l'anatomie, le taux d'hypertrophie manifesté par les animaux.

L'origine de la différence constatée entre régions se trouve dans les différences de masse des muscles qui les composent (tabl. 2). Il est particulièrement net qu'on enregistre un développement considérablement plus marqué de la majeure partie des muscles du membre postérieur.

Au niveau des muscles il n'est pas établi, non plus, d'hypertrophie généralisée, mais une hypertrophie différentielle qui paraît affecter davantage la périphérie de la musculature ; ce résultat est en accord avec les résultats de POMEROY et WIILIAMS (I962). Cette observation semble infirmer 1'hypothèse de Vissac (I968) selon laquelle le gradient de la manifestation du caractère culard serait céphalocaudal, ou celle de 
Rollins et al. (r969) pour lesquels le degré d'expression de l'hypertrophie se manifesterait selon un gradient proximo-distal.

I,es muscles où 1'hypertrophie est la plus importante sont ceux qui présentent une face importante en plan superficiel, tels que les muscles Cutaneus trunci, Latissimus dorsi, Pectoralis profundus, Triceps brachii (Caput laterale). Les muscles de la cuisse, imbriqués les uns dans les autres, mais offrant une face externe superficielle, présentent une hypertrophie de Io à I4 p. IOo. Les muscles dont le développement

\section{TABLEAU 2}

Masse (en g) des principaux muscles de la carcasse et leur développement relatif chez les animaux hypertrophiés

$$
\frac{\mathrm{H}-\mathrm{N}}{\mathrm{N}} \times \text { 100 }
$$

\begin{tabular}{|c|c|c|c|c|}
\hline Muscles & $\mathrm{H}$ & $\mathbf{N}$ & $\frac{\mathrm{H}-\mathrm{N}}{\mathrm{N}}$ & $-N \times 100$ \\
\hline Cutaneus trunci & 3346 & 2493 & & 34,22 \\
\hline Tensor fasciae latae ............ & 2198 & 1827 & & 20,31 \\
\hline Latissimus dorsi $\ldots \ldots \ldots \ldots \ldots$ & 3553 & 3028 & & 17,34 \\
\hline Triceps brachii caput laterale ..... & 931 & 798 & & 16,67 \\
\hline Pectoralis profundus .......... & 6264 & $5 \lcm{419}$ & & 15,59 \\
\hline Semi membranosus ........... & 7151 & 6239 & & 14,62 \\
\hline Vastus lateralis .............. & 3564 & 3127 & & 13,98 \\
\hline Biceps femoris $\ldots \ldots \ldots \ldots \ldots \ldots$ & 11200 & 10029 & & 11,68 \\
\hline Semi tendinosus .. & 3610 & 3282 & & 9,99 \\
\hline Trapezius $\ldots \ldots \ldots \ldots \ldots \ldots$ & 2165 & 2005 & & 7,98 \\
\hline Rectus femoris ............. & 3059 & 2847 & & 7,45 \\
\hline Triceps brachii caput longum ..... & 4945 & 4652 & & 6,30 \\
\hline Pectineus ................... & 814 & 772 & & 5,44 \\
\hline Gracilis .................. & 1932 & 1836 & & 5,23 \\
\hline Psoas major ................. & 2421 & 2305 & & 5,03 \\
\hline Teres major .................. & 616 & 594 & & 3,70 \\
\hline Obliquus externus abdominis ..... & 3136 & 3068 & & 2,22 \\
\hline 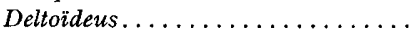 & 754 & 741 & & 1,75 \\
\hline Gastrocnemius ............. & 2649 & 2669 & - & 0,75 \\
\hline Longissimus dorsi $\ldots \ldots \ldots \ldots$ & 9350 & 9447 & 一 & 1,03 \\
\hline Adductor femoris ............ & 2220 & 2245 & - & 1,11 \\
\hline Brachialis $\ldots \ldots \ldots \ldots \ldots \ldots \ldots$ & 552 & 564 & 一 & 2,13 \\
\hline Rhomboïdeus.............. & 2237 & 2288 & - & 2,23 \\
\hline Supra spinatus ............. & 1966 & 2013 & - & 2,34 \\
\hline Gluteus medius . ............. & 5196 & 5332 & 一 & 2,55 \\
\hline Gluteus profundus ............ & 320 & 339 & $\longrightarrow$ & 5,60 \\
\hline Biceps brachii $\ldots \ldots \ldots \ldots \ldots \ldots$ & 736 & 796 & 一 & 7,54 \\
\hline Infra spinatus $\ldots \ldots \ldots \ldots \ldots$ & 2598 & 2811 & - & 7,58 \\
\hline Sub scapularis.............. & 1438 & 1591 & 一 & 9,62 \\
\hline Transversus abdominis ........ & 1883 & 2087 & - & 9,77 \\
\hline Rectus abdominis ........ & 2928 & 3259 & - & 10,16 \\
\hline Iliacus $\ldots \ldots \ldots \ldots \ldots \ldots \ldots \ldots$ & 954 & 1125 & 一 & 15,20 \\
\hline 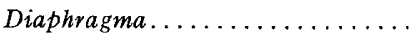 & 726 & 885 & - & 17,97 \\
\hline Obliquus internus abdominis ..... & 1350 & 1670 & 一 & 19,16 \\
\hline Teres minor $\ldots \ldots \ldots \ldots \ldots \ldots$ & 176 & 220 & - & 20,00 \\
\hline Semi spinalis capitis .......... & 2097 & 2836 & - & 26,06 \\
\hline 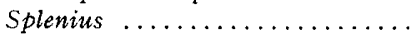 & 1369 & 1970 & 一 & 30,51 \\
\hline Vastus medialis ......... & 1073 & 1729 & - & 37,94 \\
\hline
\end{tabular}


relatif est inférieur chez le type "culard " par rapport au type " normal " se situent en plan profond, tels l'Iliacus (- I5,20 p. I0o), Subscapularis ( $-9,62$ p. I00), Gluteus profundus (- 5,60 p. Iоo).

Dans l'encolure des animaux de type hypertrophié les muscles Semispinalis capitis et Splenius marquent un net sous-développement. Les deux muscles en question se situent en plan profond, ce qui est conforme à l'hypothèse de l'hypertrophie superficielle. Leur moindre importance pourrait être également la conséquence d'un retard du développement des caractères sexuels secondaires. Les muscles Semispinalis capitis et Splenius participent normalement, chez le mâle, à l'épaississement de l'encolure, caractère sexuel secondaire des taureaux. Leur moindre développement dans le type " culard " pourrait étayer l'hypothèse d'une immaturité sexuelle, au stade d'examen considéré, que suggèrent d'autres observations faites dans la pratique de l'élevage (faible développement de l'encornage, difficultés de saillies dues à un moindre développement génital, fertilité réduite).

D'autre part, les muscles intervenant dans la mécanique des phénomènes respiratoires (Diaphragma et muscles de la région thoracique costale) manifestent une nette tendance au sous-développement, dont les conséquences sur le plan de l'intensité de la respiration pourraient conduire chez le type hypertrophié à une hypoventilation contribuant, en partie, à un état d'hypoxie.

Parmi les muscles à fort degré d'hypertrophie on doit considérer avec intérêt le muscle Triceps brachii (Caput laterale), dont la localisation anatomique autorise un prélèvement facile et peu onéreux. On pourrait s'en servir pour définir un indice objectif de mesure de l'hypertrophie musculaire en rapportant sa masse soit au poids de la carcasse, soit mieux, à l'un des éléments du squelette, l'os canon par exemple.

\section{Rapport entre la musculature et le squelette}

Le tableau 3 indique la valeur des estimations de la masse de 1'ensemble des os de la demi-carcasse et celle des os des membres, à poids de muscle comparable ( $140 \mathrm{~kg}$ ) dans le type normal et le type hypertrophié ; on doit noter chez ce dernier une réduction importante du squelette qui est en accord avec les résultats des autres auteurs (BUTTERFIELD, I966 ; VisSAC, I968). Cette réduction entraîne des différences sensibles dans la valeur du rapport muscle/os de l'ensemble du corps et des différentes régions (tabl. 4). La variation du rapport, d'une région à l'autre, se superpose à celle précédemment constatée pour les muscles, le pourcentage des différents segments osseux des membres étant voisin dans les deux types. La valeur du rapport muscle/os observé sur le matériel animal de l'expérience correspond à la valeur la plus élevée qui ait jamais été citée, à notre connaissance, pour ce rapport. On peut estimer qu'elle approche la valeur limite biologique de ce rapport au-delà de laquelle les animaux ne peuvent vraisemblablement présenter une viabilité suffisante, au plan zootechnique tout du moins (Dumont et BOCCARD, I967).

\section{Composition de la musculature}

Le tableau 5 indique les résultats concernant la teneur en collagène des muscles exprimée par la valeur du rapport

$$
\frac{\text { azote de 1'hydroxyproline }}{\text { azote total }} \times 10^{3}
$$


Les muscles sont classés dans l'ordre décroissant de l'hypertrophie observée ci-dessus. A une exception près ( $\mathrm{m}$, Semispinalis capitis), muscle situé dans la région cervicale dont nous avons déjà évoqué 1'hypotrophie, tous les muscles étudiés présentent, chez le type " hypertrophié " un déficit de collagène. Les résultats sont en accord avec ceux de LAWRIE et al. (I964) qui, sur I4 muscles de génisse, ont trouvé des teneurs en hydroxyproline inférieures chez la génisse hypertrophiée.

\section{TABLEAU 3}

Importance de l'ossature

(à I $40 \mathrm{~kg}$ de muscles dans la demi-carcasse)

\begin{tabular}{|c|c|c|c|c|}
\hline & \multicolumn{2}{|c|}{ Type normal } & \multicolumn{2}{|c|}{ Type hypertrophié } \\
\hline & $\begin{array}{c}\text { Masse } \\
\text { (g) }\end{array}$ & $\begin{array}{l}\text { Pourcentage } \\
\text { du squelette }\end{array}$ & $\begin{array}{l}\text { Masse } \\
\text { (g) }\end{array}$ & $\begin{array}{l}\text { Pourcentage } \\
\text { du squelette }\end{array}$ \\
\hline $\begin{array}{l}\text { Ensemble des os } \\
\text { de la demi-carcasse }\end{array}$ & 26410 & 100 & 20156 & 100 \\
\hline Omoplate $\ldots . . \ldots$. & 1359 & 5,15 & 1036 & 5,14 \\
\hline Humérus ......... & 2206 & 8,56 & 1719 & 8,53 \\
\hline Radius + Cubitus ... & 2178 & 8,25 & 1882 & 9,34 \\
\hline Bassin $\ldots \ldots \ldots \ldots$ & 2106 & 7,97 & 1561 & 7,75 \\
\hline Fémur........... & 2901 & 10,98 & 2208 & 10,95 \\
\hline Tibia $\ldots \ldots \ldots \ldots$ & 3215 & 12,17 & 2616 & 12,98 \\
\hline
\end{tabular}

TABLEAU 4

Valeur du rapport musclelos

\begin{tabular}{|c|c|c|}
\hline Région anatomique & Type normal & Type hypertrophié \\
\hline $\begin{array}{c}\text { Ensemble } \\
\text { de la demi-carcasse }\end{array}$ & 5,30 & 6,95 \\
\hline $\begin{array}{l}\text { Membre antérieur } \\
\text { dans son ensemble }\end{array}$ & 3,27 & 4,02 \\
\hline Épaule ......... & 6,02 & 7,44 \\
\hline Bras $\ldots \ldots \ldots \ldots \ldots \ldots \ldots$ & 3,28 & 4,41 \\
\hline Avant-bras $\ldots \ldots \ldots \ldots$ & 1,54 & 1,77 \\
\hline $\begin{array}{l}\text { Membre postérieur } \\
\text { dans son ensemble }\end{array}$ & 5,83 & 7,91 \\
\hline Croupe ...... & 3,31 & 4,22 \\
\hline Cuisse $\ldots \ldots \ldots \ldots \ldots \ldots$ & 11,93 & 17,23 \\
\hline Jambe $\ldots \ldots \ldots \ldots \ldots$ & 1,97 & 2,26 \\
\hline
\end{tabular}


On doit constater que le déficit relatif en collagène des muscles de type hypertrophié est indépendant de leur degré de développement pondéral défini par $\frac{\mathrm{H}-\mathrm{N}}{\mathrm{N}} \times$ IOO.

\section{TABLEAU 5}

Variation de la teneur en collagène

(N Hydroxyproline/ $\mathrm{N}$ total $\times \mathrm{IO}^{3}$ ) de différents muscles

\begin{tabular}{|c|c|c|c|c|}
\hline \multirow[b]{2}{*}{ Muscles } & \multirow{2}{*}{$\begin{array}{c}\text { Degré } \\
\text { d'hypertrophie } \\
\frac{\mathrm{H}-\mathrm{N}}{\mathrm{N}} \times 100\end{array}$} & \multicolumn{2}{|c|}{ Teneur en collagène } & \multirow[b]{2}{*}{$\frac{\mathrm{C}_{\mathrm{H}}-\mathrm{C}_{\mathrm{N}}}{\mathrm{C}_{\mathrm{N}}} \times 100$} \\
\hline & & $\begin{array}{c}\text { Type } \\
\text { hypertrophié } \\
\mathrm{C}_{\mathbf{H}}\end{array}$ & $\begin{array}{l}\text { Type normal } \\
\mathrm{C}_{\mathrm{N}}\end{array}$ & \\
\hline Latissimus dorsi & 17,34 & 2,295 & 3,280 & $-30,03$ \\
\hline Triceps brachii caput laterale & 16,67 & 2,250 & 5,145 & $-56,26$ \\
\hline Pectoralis profundus ......... & 15,59 & 3,265 & 4,245 & $-23,09$ \\
\hline Semitendinosus ............ & 9,99 & 2,270 & 3,455 & $-34,30$ \\
\hline Triceps brachii Caput longum ... & 6,30 & 2,060 & 2,675 & $-24,00$ \\
\hline 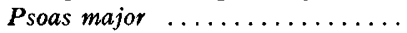 & 5,03 & 0,900 & 1,255 & $-28,29$ \\
\hline Teres major . . . . . . . . . . . & 3,70 & 1,150 & 2,085 & $-44,84$ \\
\hline Longissimus dorsi ....... & $-1,03$ & 1,650 & 2,165 & $-23,79$ \\
\hline Brachialis ............... & $-\quad 2,13$ & 2,945 & 3,654 & $-19,20$ \\
\hline Rhomboïdeus ............. & $-2,23$ & 2,510 & 3,600 & $-30,28$ \\
\hline Supra spinatus ............ & $-\quad 2,34$ & 2,185 & 3,925 & $-44,33$ \\
\hline Infra spinatus $\ldots \ldots \ldots \ldots \ldots$ & $-7,58$ & 3,195 & 4,075 & $-21,60$ \\
\hline Semispinalis capitis .......... & $-26,06$ & 2,910 & 2,885 & $+00,86$ \\
\hline
\end{tabular}

\section{CONCLUSION}

\section{Hypothèse sur les causes de l'origine de l'hypertrophie musculaire héréditaire des bovins}

Sur le plan de l'anatomie musculaire l'animal hypertrophié se distingue du type normal de la même race et du même sexe par les deux caractéristiques suivantes :

- une insuffisance très nette de l'équipement en collagène de l'appareil locomoteur : la masse osseuse est réduite, pour un même poids de muscle, et la teneur en collagène des muscles est sensiblement abaissée ;

- une hypertrophie différentielle et périphérique de la musculature. Il a été établi d'autre part que chez le bovin hypertrophié la trame du tissu conjonctif intramusculaire présente un aspect lâche caractéristique (Dumont et SchmitT, I973).

Il peut être intéressant de relier ces différents caractères et de tenter d'en expliquer l'origine par une cause primaire commune. L'hypothèse que nous proposons dans ce but est qu'il existe au tade post natal chez l'animal de type hypertrophié une insuffisance de la capacité respiratoire concomitante d'un sous-développement des muscles de la respiration et des organes respiratoires (le poumon du type hypertrophié étant moins lourd que celui de l'animal normal). Cette insuffisance ralentirait 
la synthèse du collagène pour l'élaboration duquel l'oxygène respiratoire est indispensable (Fujimoto et TAMIYA, I962), le déficit de collagène se répercuterait par un amoindrissement général des tensions auxquelles sont normalement soumis, dans leur développement, les muscles au cours de la croissance, qu'il s'agisse des contraintes externes que représentent la peau, les fascia ainsi que les aponévroses, ou qu'il s'agisse des contraintes internes résultant de la présence du périmysium infiltrant chacun des muscles. La réduction de la tension conjonctive serait à l'origine de l'hypertrophie. Les possibilités d'expression de la sous-tension conjonctive des faisceaux de fibres musculaires, génératrices, selon nous, d'hypertrophie seraient naturellement limitées par les contraintes externes propres à chacun des muscles, et par conséquent, très variables d'un muscle à l'autre.

Ainsi pourrait-on expliquer $\mathrm{I}^{\mathrm{O}}$ ) le fait que le degré de développement pondéral ou volumique du muscle soit indépendant de son déficit en collagène intramusculaire ; $2^{\circ}$ ) l'hypertrophie périphérique, mise en évidence par cette étude anatomique et que laisse supposer déjà l'examen des animaux. On a reconnu de longue date que les masses musculaires de bovins hypertrophiés paraissent, tant sur la carcasse que sur le sujet vivant, jaillir hors des zones de tension que représent les supports osseux et les sillons intermusculaires.

Cette hypothèse d'une insuffisance de capacité respiratoire à différents niveaux physiologiques du type hypertrophié avancée ici pour expliquer la variation enregistrée au plan de l'organisation de la musculature est corroborée par les études récentes des mécanismes physiologiques et biochimiques des phénomènes respiratoires, dans les conditions normales et à l'issus d'effort musculaire (HOLMEs, AsHMORE et Robinson, I973; Monin et BocCARD, I974).

Rę̧u pour publication en mai 1974.

\section{REMERCIEMENTS}

Nous remercions MM. Dauzier, Menissier et Ouhayoun des remarques et suggestions qu'ils ont formulées à la lecture du manuscrit.

\section{SUMMARY}

\section{CONSEQUENCES OF HEREDITARY MUSCULAR HYPERTROPHY ON MUSCLES IN CATTLE}

The muscles from half carcasses of charolais bull, 9 with muscular hypertrophy and $\mathbf{I} 7$ normal and aged between 10 and 20 months, were dissected anatomically. Using regression equations, the weights of the main muscles were estimated at a mean total muscle weight of $140 \mathrm{~kg}$.

The comparison between the two groups of animals was made on the basis of the ratio $\frac{\mathrm{H}-\mathrm{N}}{\mathrm{N}} \times$ roo where $\mathrm{H}$ is the weight of one muscle or group of muscles in hypertrophied animals and $\mathbf{N}$ the same component in normal animals.

At constant total muscle weight the hypertrophy affects the crural $(+9.92 \mathrm{p}$. Ioo) thoracic $(+6.77$ p. I0o) brachial $(+5,02$ p. IOo) parts only, the other anatomical parts being variously delayed in their development. 
The observed modifications in muscle distribution in muscular hypertrophy explain changes in appearance and conformation of the live animal.

Hypertrophy mainly affects muscles in the thigh (Tensor fasciae latae : $+20,31$; Vastus lateralis : + 13.98; Semimembranosus : + 14.62; Biceps femoris : + I1.68; Semitendinosus : +9.99 ; Rectus femoris $:+7.45$; Gracilis $:+5.23$; Pectineus $:+5.44$ p. 10o), and in other regions superficial muscles only (Latissimus dorsi : +17.34 ; Pectoralis profundus : +15.59 ; Triceps brachii caput laterale $:+16.67$ and Trapezius : +7.98 p. 100).

The following muscles are less developed at the same total muscle weight in hypertrophied animals than in normal animals :

- muscles of the abdominal wall,

- muscles of the upper part of the neck, probably related to the sexual immaturity of these animals,

- muscles in connection with the respiratory system (Diaphragma:- 17.97 ; intercostal : - II.93 p. 100).

The collagen content of muscles of hypertrophied animals is less than that of normal animals and is independent of the relative development of these muscles.

A hypothesis concerning the origin of muscular hypertrophy is proposed in which the hypertrophy of the peripheral muscles is considered to be the consequence of the general collagen deficit which allows these muscles to expand, and the collagen deficit itself is considered to be the consequence of the low respiratory capacity which is one of the characteristics of animals with muscular hypertrophy.

\section{RÉFÉRENCES BIBLIOGRAPHIQUES}

Angervall L., Carlstrom E., I963. Theoretical criteria for the use of relative organ weights and similar ratios in biology. J. Theoretical Biol., 4, 254-259.

Boccard R., Dumont B. L., r96o. Étude de la production de la viande chez les ovins. II. Variation de l'importance relative des différentes régions corporelles de l'agneau de boucherie. Ann. Zootech., 9, $355-363$.

Boccard R., I968. Variation de la teneur en hydroxyproline des muscles de porcs Large White et Piétrain. Ann. Zootechn., 17, 7I-75.

Butterfield R. M., I966. Muscular hypertrophy of cattle. Aust. Vet. J., 42, 37-39.

Dumont B. L., Boccard R., I967. Le rapport muscle/os, critère de sélection des bovins de boucherie. II Symp. inter. Zootechn., Milano, r49-155.

Dumont B. L., Sснмiтt O., 1973. Conséquences de l'hypertrophie musculaire héréditaire sur la trame conjonctive du muscle de bovin. Ann. Génét. Sél. anim., 5, 499.506.

Fujimoto D., TAмIYA N., I962. Incorporation of ${ }^{18} \mathrm{O}$ from air into hydroxyproline by chick embryo. Biochem. J., 84, 333-335.

Holmes J. H. G., Ashmore C. R., Robinson D. W., r973. Effects of stress on cattle with hereditary muscular hypertrophy. J. anim. Sci., 36, 684-694.

McKellar F. C., Ouhayoun J., I973. Étude du caractère culard. VII. Fréquence et expression en race South Devon. Ann. Génét. Sel. anim., 5, 163-176.

Lauvergne J., Vissac B., Perramon A., I963. Étude du caractère culard. I. Mise au point bibliographique. Ann. Zootech., 12, r33-I56.

Lawrie R. A., Pomeroy R. W., Williams R. P., ig64. Studies in the muscles of meat animals. IV. Comparative composition of muscles from "doppelender " and normal stbling heifer. J. agric. Sci., 62, $89-92$.

Monin G., Boccard R., I974. Caractéristiques physiologiques respiratoires des bovins culards. Ann. Génét. Sél. Anim., 6, I87-193.

Pomeroy R. W., Williams D. R., I962. Muscular hypertrophy in cattle (Abstract). Anim. Prod., 4, 302 .

Rollins W. C., Julian L. M., Carroll F. D., rg69. A note on the body composition of a double muscled female and a normal female from a line bred Aberdeen Angus herd. Anim. Prod., 11, III-II4.

Rollins W. C., Tanaka M., Nott C. F. G., Thiessen R. B., I972. On the mode of inheritance of double-muscled conformation in bovines. Hilgardia, 41, 433-456.

Vissac B., 1968. Étude du caractère culard. II. Incidence du caractère culard sur la morphologie générale des bovins. Ann. Zootech., 17, 77-ror. 